\title{
7 Gesetzliche Regelungen und aktueller Stand der Aktivitäten
}

\subsection{Vorbemerkung}

Der erste, unmittelbare Eindruck ist: das Gebiet Qualität und Patientensicherheit war in Deutschland in den letzten 20 Jahren ein Gebiet intensivster gesetzgeberischer Aktivitäten. Wie es gelegentlich in Einführungsreferaten zum Thema gesagt wird: ein Sitzungstag reicht kaum aus, die unterschiedlichen Maßnahmen überhaupt nur aufzuzählen. Allerdings hinterlässt diese Regelungsvielfalt auch einen etwas disparaten Eindruck, der Grad der Detailierung und die große Zahl der Einzelregelungen lässt den Interessierten zuweilen sprachlos zurück. Ohne Zweifel viel Aktivität, aber wo bleibt die verbindende Melodie?

In diesem Kapitel wird der Versuch unternommen, die bestehenden gesetzlichen Regelungen zumindest in Ausschnitten darzustellen. Die Darstellungen bedienen sich der sechs Qualitätsperspektiven aus Kapitel 1.3, die diesem Gutachten als Systematik der Sichtweisen auf das Thema Qualität und Sicherheit zugrunde gelegt wurden:

- die gesellschaftliche bzw. Populationsperspektive, repräsentiert insbesondere durch die Regelungen des SGB V Kapitel IV;

- die Perspektive des Nutzens (Allokation und Effizienz);

- die Patientenperspektive (Selbstbestimmung);

- die professionelle Perspektive (Autonomie, Garantenstellung); 
- die institutionelle Perspektive (Organisation);

- wissenschaftliche Perspektive (Deskription, Hypothesenbildung).

Es werden hierbei zusätzlich, über die gesetzlichen Regelungen hinaus, auch untergesetzliche Regelungen mit einbezogen, vor allem solche, die der Selbstverwaltung (in erster Linie Gemeinsamer Bundesausschuss) aufgegeben sind. In begrenztem Umfang werden auch professionelle und institutionelle Initiativen mit aufgeführt.

\subsection{Gesellschaftliche und Populationsperspektive}

Die gesellschaftliche und Populationsperspektive umfasst den großen Bereich der genuinen Qualitätsaspekte, aber auch umgrenztere Themen wie nosokomiale Infektionen und Patientensicherheit. Die Tatsache, dass die Regelungen von $\mathbb{} 135$ und Folgende im vierten Kapitel des 5. Sozialgesetzbuches unter der Überschrift „Beziehungen der Krankenkassen zu den Leistungserbringern“ untergebracht sind (9. Abschnitt „Sicherung der Qualität der Leistungserbringung"), zeigt bereits auf, dass - wie bereits in Kapitel 4.1 (s. Abb. 28) ausführlich beschrieben - es sich hier um Maßnahmen zur Qualitätssicherung handelt, die aus gesellschaftlicher Perspektive unerwünschten Effekten der vorherrschenden Vergütungssystematik vorbeugen sollen. Daher hat eine systematische externe Qualitätssicherung auch erst zusammen mit der Einführung der Sonderentgelte und Fallpauschalen im Jahr 1993 an Fahrt aufgenommen, als man besonders wegen der drohenden Verweildauerverminderung der von der Fallpauschalenregelung betroffenen Krankheitsbilder Bedenken bzgl. der Qualität hatte. Zunächst (bis zur GKV 200o-Reform) war diese Qualitätssicherung auf den Krankenhausbereich beschränkt und wurde auf Landesebene organisiert (Landesgeschäftsstellen), danach - im Rahmen der DRG-Einführung - hat sich die Bundesebene verstärkt um die Thematik bemüht, bevor im Gesundheitsmodernisierungsgesetz 2005 die Delegation an den Gemeinsamen Bundesausschuss und damit verbundene Institutionen vorgenommen wurde ( $\$ 137$ SGB V). Mittlerweile sind Maßnahmen der Qualitätssicherung und des Qualitätsmanagements auf alle Sektoren des Gesundheitssystems ausgedehnt worden. Mit dem GKV-FQWG vom 5.6.2014 ist dem GBA ein „Institut für Qualitätssicherung und Transparenz im Gesundheitswesen" zur Seite gestellt worden.

\subsubsection{Externe Qualitätssicherung und internes Qualitätsmanagement}

Die zentralen Bestimmungen finden sich in $\mathbb{1} 135 \mathrm{a}$ und $₫ 37$. Unter Bezugnahme auf $\mathbb{s} \mathbf{2}$ SGB V („Qualität und Wirtschaftlichkeit haben dem allgemeinen Stand der medizinischen Erkenntnisse zu entsprechen“ und „Krankenkassen, Leistungserbringer und Versicherte haben darauf zu achten, dass die Leistungen wirksam und wirtschaftlich erbracht werden“) in Verbindung mit $\mathbb{5} \mathbf{7 0}$ SGB V (,in der fachlich gebotenen Qualität“) werden in $\mathbb{1}$ 135a SGB V (in Ver- 
bindung mit $₫ 155 \mathrm{~b}$ [ambulantes Operieren] und $₫ 116 \mathrm{~b}$ [ambulante spezialärztliche Versorgung]) alle zugelassenen Leistungserbringer, also über alle Sektoren hinweg, zur „Sicherung und Weiterentwicklung der Qualität“ verpflichtet, die „Leistungen müssen dem jeweiligen Stand der wissenschaftlichen Erkenntnisse entsprechen und in der fachlich gebotenen Qualität erbracht werden“ ( $\$ 135 \mathrm{a}$ Abs. 1). Es werden bereits an dieser Stelle sehr deutlich die „einrichtungsübergreifenden Maßnahmen“ der Qualitätssicherung herausgehoben, „die insbesondere zum Ziel haben, die Ergebnisqualität zu verbessern“" (Abs. 2). In allen aufgeführten Einrichtungen muss ein internes Qualitätsmanagement eingeführt werden. Die einrichtungsübergreifenden Maßnahmen und das interne Qualitätsmanagement werden nach $\$ 137$ SGB V (Abs. 1 Satz 1 Nr. 1) durch Richtlinien des Gemeinsamen Bundesausschusses gemäß $₫ 92$ Abs. 1 Satz 2 Nr. 13 spezifiziert und die Teilnahme an ihnen im Sinne eines negativen Pay for Reporting (s. Kap. 4.4) sanktionsbewehrt. Die Richtlinien sollen möglichst sektorenübergreifend erlassen werden, ebenso wie die

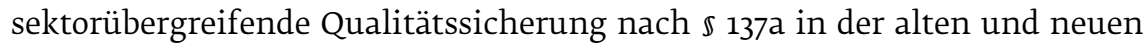
(FQWG) Fassung. Hinsichtlich der ,indikationsbezogene(n) Notwendigkeit und Qualität der durchgeführten diagnostischen und therapeutischen Leistungen, insbesondere aufwändiger medizintechnischer Leistungen" sind „Mindestanforderungen an die Struktur-, Prozess- und Ergebnisqualität festzulegen“ - im anschließenden Absatz 1a wird ähnlich wie in $\mathbb{1} 137 \mathrm{a}$ Abs. 1 Satz Nr. 1 in fachlich korrekter Form der Begriff „Indikator“ verwendet.

An dieser Stelle und in damit verbundenen Regelungen führt der Gesetzgeber erstaunlich detaillierte Regelungen $\mathrm{zu}$ (wichtigen) Teilbereichen auf:

- die Richtlinien müssen „Maßnahmen zur Sicherung der Hygiene“ einschließlich „Indikatoren zur Beurteilung der Hygienequalität“, unter Berücksichtigung von „etablierte(n) Verfahren zur Erfassung, Auswertung und Rückkopplung von nosokomialen Infektionen, antimikrobiellen Resistenzen und zum Antibiotikaverbrauch“ umfassen ( $\$ 137 \mathrm{Abs}$. 1a), die nachfolgend nach Krankenhausentgeltgesetz vom 15.7.2013 z.B. zur teilweisen Erstattung der Personalkosten von zusätzlich eingestellten Hygienefachkräften von $90 \%$ führen (Hygienefachärzte $75 \%$, s. $\mathbb{4} 4$ Abs. 11);

- diese Hygieneindikatoren „sind in den Qualitätsberichten nach Absatz 3 Nummer 4 darzustellen“, „zusätzliche Anforderungen“ für den Qualitätsbericht können formuliert werden (Abs. 1b);

- die Sektorproblematik in der MRSA-Epidemiologie wird adressiert ( $\$ 92$ Abs. 7 Satz 1 Nr. 4: „Näheres zur Verordnung häuslicher Krankenpflege zur Dekolonisation von Trägern mit dem methicillinresistenten Staphylococcus aureus (MRSA).“), indem dem GBA hier die Entwicklung von Richtlinien aufgegeben wird;

- „Maßnahmen zur Sicherung der Qualität in der psychiatrischen und psychosomatischen Versorgung" sollen festgelegt werden (im Zusammenhang zur DRG-Einführung im Bereich der Psychiatrie und Psycho- 
somatik), auch hier sollen „Indikatoren zur Beurteilung der Struktur-, Prozess- und Ergebnisqualität für die einrichtungs- und sektorenübergreifende Qualitätssicherung “ entwickelt werden (Abs. 1c);

- "wesentliche Maßnahmen zur Verbesserung der Patientensicherheit“ sollen per Richtlinie ins Auge gefasst werden, ,insbesondere Mindeststandards für Risikomanagement- und Fehlermeldesysteme“, über die in den Qualitätsberichten nach Abs. 3 informiert werden soll. Der GBA formuliert „Anforderungen an einrichtungsübergreifende Fehlermeldesysteme, die in besonderem Maße geeignet erscheinen, Risiken und Fehlerquellen in der stationären Versorgung zu erkennen, auszuwerten und zur Vermeidung unerwünschter Ereignisse beizutragen“, für die dann lt. Krankenhausfinanzierungsgesetz vom 15.7.2013 „Zuschläge zu vereinbaren “ sind, „diese können auch in die Fallpauschalen eingerechnet werden.“ ( $\$ 17$ b Abs. 1 Satz 5);

- die Richtlinien des GBA sollen gemäß $\mathbb{1} 137$ Abs. 3 Satz 1 Nr. 4 „Inhalt, Umfang und Datenformat eines jährlich zu veröffentlichenden strukturierten Qualitätsberichts der zugelassenen Krankenhäuser “ umfassen, der die Maßnahmen zur Qualitätssicherung, das interne Qualitätsmanagement, die Maßnahmen zum Risikomanagement (s. KQM-RL vom 23.1.2014: $\mathbb{5} 5$, Abs. 6 Satz 4; s.u.), die Hygieneindikatoren sowie „Art und Anzahl der Leistungen des Krankenhauses“ ausweist und „Landesverbänden der Krankenkassen und den Ersatzkassen im Internet zu veröffentlichen" ist (s. Kap. 5.1);

- weiterhin sollen die Richtlinien Fortbildungspflichten für Fachärzte (s. Kap. 7.5) und

- „Grundsätze zur Einholung von Zweitmeinungen“ umfassen;

- außerdem sollen die Richtlinien Angaben zum Qualitätsindikator Mindestmengen enthalten, die wenn sie „voraussichtlich“ nicht erreicht werden, nicht erbracht werden dürfen ( $\$ 137$ Abs. 3 Satz 1 Nr. 2 und Satz 2) (s. Kap. 3.2.3 sowie 4.2), wenn vonseiten der Länder keine Ausnahmeregelungen erteilt werden.

Der GBA ist der Aufforderung zur Entwicklung der entsprechenden Richtlinien nachgekommen:

- In der „Richtlinie über Maßnahmen der Qualitätssicherung in Krankenhäusern" (QSKH-RL) in der Fassung vom 20.7.2013 (in Kraft seit 1.1.2014) (GBA 2014A) werden vom GBA die Maßnahmen zur externen Qualitätssicherung nach $\mathbb{1} 137$ Abs. 1 spezifiziert. In $\mathbb{2} 2$ werden als ,Ziele der Qualitätssicherung" genannt,

a) „durch Erkenntnisse über Qualitätsdefizite Leistungsbereiche systematisch zu identifizieren, für die Qualitätsverbesserungen erforderlich sind.

b) Unterstützung zur systematischen, kontinuierlichen und berufsgruppenübergreifenden einrichtungsinternen Qualitätssicherung (internes Qualitätsmanagement) zu geben. 
c) Vergleichbarkeit von Behandlungsergebnissen, insbesondere durch die Entwicklung von Indikatoren, herzustellen.

d) durch signifikante, valide und vergleichbare Erkenntnisse, insbesondere zu folgenden Aspekten, die Qualität von Krankenhausleistungen zu sichern:

- Indikationsstellung für die Leistungserbringung,

- Angemessenheit der Leistung,

- Erfüllung der strukturellen und sächlichen Voraussetzungen zur Erbringung der Leistungen,

- Ergebnisqualität.“

Diese Ziele werden als relativ verbindlich dargestellt, denn „die leitende Ärztin oder der leitende Arzt der Fachabteilung [ist] verpflichtet, die Ergebnisse, Vergleiche und Bewertungen, die ihr oder ihm aus der Qualitätssicherung zugeleitet werden, mit allen Mitgliedern der Krankenhausleitung und den unmittelbar am Behandlungsprozess beteiligten Mitarbeiterinnen und Mitarbeitern anhand der Ergebnisse der Qualitätssicherungsmaßnahme durchzusprechen, kritisch zu analysieren und ggf. notwendige Konsequenzen festzulegen." (\$ 3 Abs. 2), über diese Gespräche ist ein Nachweis zu führen (Abs. 3). Analog zu den Regelungen im Infektionsschutzgesetz $(\$ 23)$ sind diese Regelungen vor allem im Fall eines unerwünschten Ereignisses mit juristischen Implikationen als relevant anzusehen. Die Richtlinie beschreibt Datenerhebung, Datenfluss zwischen den verschiedenen Ebenen, legt den Level des Public Reportings fest (nämlich auf dessen untersten Stufe mit Anonymisierung der Wettbewerber, s. Kap. 5.1). Die Daten werden einmal jährlich veröffentlicht, zur Validierung wird ein Stichprobenverfahren mit Zweitbegutachtung angewandt ( $5 \%$ der Krankenhäuser), bei Auffälligkeiten wird ein sog. Strukturierter Dialog eingeleitet, der bis hin zu einer Begehung vorangetrieben werden kann.

- Die Richtlinie „über die einrichtungs- und sektorübergreifenden Maßnahmen der Qualitätssicherung“ (Qesue-RL, GBA 2010) bezieht sich, wie der Name schon sagt, auf die sektorübergreifenden Maßnahmen nach $\mathbb{1} 137$ Abs. 2. und betrifft daher nicht nur Krankenhäuser, sondern auch die angrenzenden Sektoren wie z.B. den ambulanten Bereich. Die Anforderungen an den Datenfluss sind hier besonders hoch. Der Auftrag der sektorenübergreifenden Perspektive findet sich auch in $\$ 137 \mathrm{a}$ bei der Aufgabenstellung der „unabhängige Institution“ ”zw. in der Fassung des FQWG vom 5.6.2014 für das „Institut für Qualitätssicherung und Transparenz im Gesundheitswesen“ (s. Kap. 7.2.3, zur Problematik des Begriffs „sektorübergreifend“ s. Kap. 2.4.8 und 9).

- Am 23.1.2014 wurde die „Qualitätsmanagementrichtlinie Krankenhäuser“ beschlossen (GBA 2014A), die die grundsätzlichen Anforderungen an das interne Qualitätsmanagement unter Einbeziehung der Patientensicherheit bzw. des Risikomanagements enthält (s. Kap. 4.3). Dieses sehr explizite Dokument steht in der Tradition des prozessorientierten 
Total Quality Management-Gedankens und nennt in der Präambel neun Grundsätze, zu denen neben Patientenorientierung, Verantwortung und Führung sowie Wirtschaftlichkeit auch Patientensicherheit sowie „Fehlervermeidung und Umgang mit Fehlern“ gehören; im Vergleich zur Fassung vom 1.10.2002 ist der Terminus „Patientensicherheit“ neu aufgenommen worden. Der Bezug zum „Qualitätswettbewerb“ (s. Kap. 4.4) wird deutlich herausgestellt ( $\$ 1$ Abs. 1 Satz 3), die externe Qualitätssicherung wird einbezogen ( $\$ 1$ Abs. 4), Doppelstrukturen zwischen Qualitäts- und Risikomanagement sollen vermieden werden (integriertes Modell, s. $\$ 5$ Abs. 2 Satz 5). Während zur genaueren Gestaltung des Qualitätsmanagement nur Vorschläge gemacht werden, wird die Einrichtung eines Risikomanagements als verpflichtend angesehen: „Das Krankenhaus hat wesentliche Maßnahmen zur Weiterentwicklung der Patientensicherheit ein- und durchzuführen.“( $\$ 5$ Abs. 1 Satz 1), „ein Fehlermeldesystem muss für alle Mitarbeiter abteilungs- und berufsgruppenübergreifend niederschwellig zugänglich und einfach zu bewerkstelligen sein“ (\$ 5 Abs. 3 Satz 1). Für die Finanzierung (verpflichtender) einrichtungsübergreifender Fehlermeldesysteme sollen Zuschläge vereinbart werden ( $\$ 5$ Abs. 6 ).

Mit dem Patientenrechtegesetz vom 11.1.2013 kam es zu einer für das Risikomanagement wichtigen Ergänzung des $\$ 135 a$ (Abs. 3), in der der Umgang mit CIRS-Meldungen thematisiert wurde:

„Meldungen und Daten aus einrichtungsinternen und einrichtungsübergreifenden Risikomanagement- und Fehlermeldesystemen nach Absatz 2 in Verbindung mit \$137 Absatz 1 d dürfen im Rechtsverkehr nicht zum Nachteil des Meldenden verwendet werden. Dies gilt nicht, soweit die Verwendung zur Verfolgung einer Straftat, die im Höchstmaß mit mehr als fünf Jahren Freiheitsstrafe bedroht ist und auch im Einzelfall besonders schwerwiegt, erforderlich ist und die Erforschung des Sachverhalts oder die Ermittlung des Aufenthaltsorts des Beschuldigten auf andere Weise aussichtslos oder wesentlich erschwert wäre.“

Diese grundsätzlich zu begrüßende Regelung enthält hinsichtlich des Strafmaßes von 5 Jahren und den „Einzelfall“ allerdings eine schwerwiegende Relativierung.

Für die zahlreichen Versorgungsformen mit Integrationscharakter (zur Übersicht s. Abb. 18) gibt es Einzelbestimmungen:

- nach $\$ 137$ f Abs. 2 Nr. 2 beschließt der GBA die bei strukturierten Behandlungsprogrammen (Disease Management) durchzuführenden Qualitätssicherungsmaßnahmen per Richtlinie:

v für die Integrierte Versorgung nach $\$ 140 \mathrm{ff}$. müssen sich lt. $\$ 140 \mathrm{ob}$ Abs. 3 „die Vertragspartner der Krankenkassen zu einer qualitätsgesicherten, wirksamen, ausreichenden, zweckmäßigen und wirtschaftlichen Versorgung der Versicherten verpflichten“; 
- für die Ambulante Spezialfachärztliche Versorgung nach $\$ 116 \mathrm{~b}$ sind lt. Richtlinie des GBA vom 21.3.2013 die nach den „Richtlinien des Gemeinsamen Bundesausschusses festgelegten einrichtungsübergreifenden Maßnahmen der Qualitätssicherung und die für den Krankenhausbereich einerseits und den vertragsärztlichen Bereich andererseits festgelegten Anforderungen an ein einrichtungsinternes Qualitätsma-

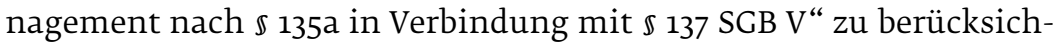
tigen;

- für das Ambulante Operieren sind nach $\$ 115 \mathrm{~b}$ Abs. 1 Satz 3 die „Qualitätsvoraussetzungen nach $\mathbb{1} 135$ Abs. 2 sowie die Richtlinien und Beschlüsse des Gemeinsamen Bundesausschusses nach $\mathbb{5} 92$ Abs. 1 Satz 2

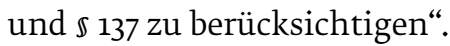

Auch im Hinblick auf Pay for Performance gab es mit dem Pflegeweiterentwicklungsgesetz vom 28.5.20o8 eine Ergänzung des $\mathbb{1} 136$, in dem Absatz 4 mit folgendem Wortlaut eingefügt wurde:

„Zur Förderung der Qualität der vertragsärztlichen Versorgung können die Kassenärztlichen Vereinigungen mit einzelnen Krankenkassen (...) ab dem 1. Januar 2009 gesamtvertragliche Vereinbarungen schließen, in denen für bestimmte Leistungen einheitlich strukturierte und elektronisch dokumentierte besondere Leistungs-, Strukturoder Qualitätsmerkmale festgelegt werden, bei deren Erfüllung die an dem jeweiligen Vertrag teilnehmenden Ärzte Zuschläge zu den Vergütungen erhalten.“

\subsubsection{Der Gemeinsame Bundesausschuss}

Die Einrichtung des Gemeinsamen Bundesausschusses (GBA) hat das deutsche Gesundheitswesen in den letzten Jahren sehr stark geprägt und wird es auch in Zukunft stark prägen; in Teilen geht dies bereits aus den in Kapitel 7.2.1 genannten Aufgaben hervor. Der GBA stellt aus politiktheoretischer Sicht eine Institutionalisierung der Ebene der Verhandlungsdemokratie dar, wenn man der Dreiteilung in Parteien, Verhandlungs- und Mediendemokratie folgen möchte (s. Korte 2012). Im Sinne einer Betonung institutioneller Elemente im Rahmen der Neokorporatismusdebatte (Lauth und Thiery 2012), erscheint der GBA als privat organisierte Struktur, die zusammen mit staatlichen und zivilgesellschaftlichen Einrichtungen die notwendigen Koordinationsaufgaben einer modernen Governance (Benz und Dose 2010, Mayntz 2010) bewerkstelligen. Aus einer mehr organisationstheoretischen Perspektive rückt noch ein anderer Gesichtspunkt in das Blickfeld, nämlich die hochgradige Autonomie der Akteure, wie sie Mintzberg wohlgemerkt auf organisatorischer Ebene bereits 1979 als professional bureaucracy (Expertenorganisation) beschrieben hat (Mintzberg 1979). Diese Autonomie bedeutet eine weitgehende Unabhängigkeit von hierarchischen Strukturen und verstärkt die ohnehin gerade im Gesundheitswesen dominante Systemkomplexität (IOM 2001; zum Arbeitsbegriff der komplexen professionellen Systembürokratie s. Kap. 8.3.3). 
Auf diese Funktion des GBA, der mit dem Gesundheitsmodernisierungsgesetz 2004 nach $\$ 91$ SGB V eingerichtet wurde, weist auch die Namensgebung der Vorgängerstruktur hin, die unter der Bezeichnung „Koordinierungsausschuss “ in $\$ 137$ e der GKV 2000-Reform vom 19.12.1999 aus den vorbestehenden Bundesausschüssen und dem Ausschuss Krankenhaus gebildet wurde. Neben drei unparteiischen Vorsitzenden ist die Zusammensetzung zwischen Leistungserbringern und Kostenträgern paritätisch geregelt. Die Beschlussregelungen weisen einen deutlichen Konsenszwang auf, insbesondere bei Leistungsausschluss. Die Konsultationspflichten mit betroffenen Einrichtungen und Verbänden sind höchst ausgedehnt, neben den pharmazeutischen Herstellern sind die Arzneimittelkommission der Deutschen Ärzteschaft ( $\$ 92 \mathrm{Abs}$. 2a) und die wissenschaftlichen Fachgesellschaften (Abs. 7a) namentlich aufgeführt. Die Beschlüsse müssen dem Bundesministerium für Gesundheit zur Genehmigung vorgelegt werden (\$94).

Die Richtlinien nach $\mathbb{5} 92$ Abs. 1 Satz 2 betreffen im Hinblick auf Qualität die Einführung neuer Untersuchungs- und Behandlungsmethoden (Nr. 5), die Arzneiverordnung (Nr. 6), die Bedarfsplanung (Nr. 9) und die Qualitätssicherung (Nr. 13). Die wichtigsten Richtlinien sind

v die Richtlinie des Gemeinsamen Bundesausschusses über die grundsätzlichen Anforderungen an ein einrichtungsinternes Qualitätsmanagement für nach $\mathbb{} 108$ SGB V zugelassene Krankenhäuser (Qualitätsmanagement-Richtlinie Krankenhäuser - KQM-RL) in der Fassung vom 21. Juni 2005, zuletzt geändert am 23. Januar 2014, in Kraft getreten am 17. April 2014 (GBA 2014A);

- die Richtlinie des Gemeinsamen Bundesausschusses nach $\$ 92$ Abs. 1 Satz 2 Nr. 13 i.V.m. $\$ 137$ Abs. 1 Nr. 1 SGB V über die einrichtungs- und sektorübergreifenden Maßnahmen der Qualitätssicherung (QesueRL) in der Fassung vom 19. April 2010, in Kraft getreten am 2. Dezember 2010; zuletzt geändert am 20. Mai 2010 veröffentlicht im Bundesanzeiger 2010 (S. 4.000) in Kraft getreten am 2. Dezember 2010 (GBA 2010);

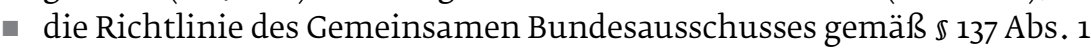
SGB V i.V.m. $\mathbb{S} 135$ a SGB V über Maßnahmen der Qualitätssicherung für nach $\$ 108$ SGB V zugelassene Krankenhäuser (Richtlinie über Maßnahmen der Qualitätssicherung in Krankenhäusern - QSKH-RL) in der Fassung vom 15. August 2006, zuletzt geändert am 20. Juni 2013, in Kraft getreten am 1. Januar 2014 (GBA 2013);

- die Richtlinie des Cemeinsamen Bundesausschusses über grundsätzliche Anforderungen an ein einrichtungsinternes Qualitätsmanagement für die an der vertragsärztlichen Versorgung teilnehmenden Ärzte, Psychotherapeuten und medizinischen Versorgungszentren (Qualitätsmanagement-Richtlinie für die vertragsärztliche Versorgung - ÄQM-RL) in der Fassung vom 18.10.2005, zuletzt geändert am 23.1.2014, in Kraft getreten am 17.4.2014 (GBA 2014B). 
Dem GBA kommen in diesem Zusammenhang folglich zwei Hauptaufgaben $\mathrm{zu}$, neben der oben bereits beschriebenen Funktion in der klassischen, diagnose- und prozedurenbezogenen externen Qualitätssicherung stehen die auf die Nutzenbewertung von Untersuchungs- und Behandlungsmethoden gerichteten Maßnahmen. In beiderlei Hinsicht wird der GBA von einer wissenschaftlichen Institution unterstützt, der Einrichtung nach $\mathbb{1}$ 137a (derzeit wahrgenommen vom AQUA-Institut, zukünftig dem Institut für Qualitätssicherung und Transparenz im Gesundheitswesen) und dem Institut für Qualität und Wirtschaftlichkeit im Gesundheitswesen (IQWiG), das sich der Nutzenbewertung widmet ( $\$ 139$ a SGB V).

\subsubsection{Von der „unabhängigen Institution“ nach $§ 137$ a zum Institut für Qualitätssicherung und Transparenz im Gesundheitswesen}

Die „fachlich unabhängige Institution“ nach $\$ 137$ a wird vom GBA beauftragt, „Verfahren zur Messung und Darstellung der Versorgungsqualität (...) zu entwickeln, die möglichst sektorenübergreifend anzulegen sind“ (Abs. 1), insbesondere „für die Messung und Darstellung der Versorgungsqualität möglichst sektorenübergreifend abgestimmte Indikatoren und Instrumente zu entwickeln“ und „die Ergebnisse der Qualitätssicherungsmaßnahmen durch die Institution in geeigneter Weise und in einer für die Allgemeinheit verständlichen Form zu veröffentlichen." (Abs. 2)

In diesem Sinne hat bis 2008 die Bundesgeschäftsstelle Qualitätssicherung (BQS) und seitdem das AQUA-Institut einen jährlichen Bericht zur Qualitätssicherung nach $\$ 137$ herausgegeben, die - bei aller Kritik über das Spektrum zu den besten Quellen für die angesprochenen Erkrankungen gehören, auch im internationalen Vergleich (zuletzt AQUA 2013). Es gibt hierzu auch eine Auswertung auf Bundeslandebene, die Einblicke in die variation-Problematik gibt (s. Kap. 3.2.2).

Mit dem GKV-Finanzstruktur- und Qualitäts-Weiterentwicklungsgesetz vom 5.6.2014 (FQWG) wird die „unabhängige Institution“ in ein rechtsfähiges „Institut für Qualitätssicherung und Transparenz im Gesundheitswesen“ („ITQuiG“) weiterentwickelt, getragen von einer vom GBA gegründeten Stiftung privaten Rechts. Nach $\mathbb{1 3 7 a}$ (neu) arbeitet das Institut „im Auftrag des Gemeinsamen Bundesausschusses an Maßnahmen zur Qualitätssicherung und zur Darstellung der Versorgungsqualität im Gesundheitswesen“(Abs. 3). Nach Abs. 3, Satz 2 soll es beauftragt werden,

1. „für die Messung und Darstellung der Versorgungsqualität möglichst sektorenübergreifend abgestimmte Indikatoren und Instrumente einschließlich Module für ergänzende Patientenbefragungen zu entwickeln,

2. die notwendige Dokumentation für die einrichtungsübergreifende Qualitätssicherung unter Berücksichtigung des Gebotes der Datensparsamkeit zu entwickeln, 
3. sich an der Durchführung der einrichtungsübergreifenden Qualitätssicherung zu beteiligen und dabei, soweit erforderlich, die weiteren Einrichtungen nach Satz 3 einzubeziehen,

4. die Ergebnisse der Qualitätssicherungsmaßnahmen in geeigneter Weise und in einer für die Allgemeinheit verständlichen Form zu veröffentlichen,

5. auf der Grundlage geeigneter Daten, die in den Qualitätsberichten der Krankenhäuser veröffentlicht werden, einrichtungsbezogen vergleichende Übersichten über die Qualität in maßgeblichen Bereichen der stationären Versorgung zu erstellen und in einer für die Allgemeinheit verständlichen Form im Internet zu veröffentlichen; Ergebnisse nach Nummer 6 sollen einbezogen werden,

6. für die Weiterentwicklung der Qualitätssicherung zu ausgewählten Leistungen die Qualität der ambulanten und stationären Versorgung zusätzlich auf der Grundlage geeigneter Sozialdaten darzustellen, die dem Institut von den Krankenkassen nach $\mathbb{2} 299$ Absatz 1a auf der Grundlage von Richtlinien und Beschlüssen des Gemeinsamen Bundesausschusses übermittelt werden sowie

7. Kriterien zur Bewertung von Zertifikaten und Qualitätssiegeln, die in der ambulanten und stationären Versorgung verbreitet sind, zu entwickeln und anhand dieser Kriterien über die Aussagekraft dieser Zertifikate und Qualitätssiegel in einer für die Allgemeinheit verständlichen Form zu informieren."

Die Forderung der sektorübergreifenden Abstimmung ist unverändert, hinzugekommen ist hier gegenüber der Vorfassung des $\mathbb{\$} 137$ a die „Module für ergänzende Patientenbefragungen“ (s. Kap. 2.4.7), weiterhin die Forderung nach Einbeziehung der Sozialdaten (Nr. 6) sowie die „Bewertung von Zertifikaten und Qualitätssiegeln“(Nr. 7).

Es ist nun also die Situation eingetreten, dass das deutsche Gesundheitswesen unversehens über zwei wissenschaftliche Institute verfügt, die beide schon im Namen den Begriff „Qualität“ bzw. „Qualitätssicherung“ tragen. Nach der Beauftragung in $\$ 137 \mathrm{a}$ (IQTiC) bzw. $\$ 139 \mathrm{aff}$. (IQWiG) SGB V lässt sich die Absicht des Gesetzgebers nachvollziehen, mit den beiden Instituten zwei wichtige Qualitätsperspektiven abzudecken, und zwar die vergleichende Qualitätssicherung im Sinne der diagnose- und prozedurenbezogenen Qualitätssicherung im IQTiC und die Nutzen- bzw. Allokationsperspektive im IQWiG. Aber

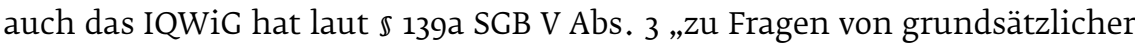
Bedeutung für die Qualität und Wirtschaftlichkeit der im Rahmen der gesetzlichen Krankenversicherung erbrachten Leistungen " tätig zu werden und dabei zur „Erstellung von wissenschaftlichen Ausarbeitungen, Gutachten und Stellungnahmen zu Fragen der Qualität und Wirtschaftlichkeit“ zu greifen sowie „für alle Bürgerinnen und Bürger verständliche(n) allgemeine(n) Informationen zur Qualität und Effizienz in der Gesundheitsversorgung “ bereitzu- 
stellen. Es wäre also nicht weit hergeholt gewesen, hätte man das IQWiG mit den Aufgaben der Qualitätssicherung betraut. In Großbritannien hat das NICE die Indikatorenentwicklung für das QOF-Programm bereits vor Jahren übernommen. Es bleibt nun die Problematik einer optimalen Abstimmung der beiden Institute durch den Gemeinsamen Bundesausschuss (s. Empfehlung 15). Dies ist besonders deswegen wichtig, weil abzusehen ist, dass beide Institute gegeneinander ausgespielt werden. Bei ausbleibendem Nutzennachweis wird man versuchen, über die „Qualitätsschiene“ zu einer Präjudizierung der Nutzenbewertung zu kommen, und bei Methoden mit nachgewiesenem Zusatznutzen werden Qualitätsanforderungen als zweitrangig dargestellt werden: eine neue Behandlungsmethode kann „richtig“ sein (Nutzennachweis), sie kann richtig angewendet werden (Qualität) oder im Idealfall natürlich beides. Dies setzt aber eine gute Koordination der Arbeit beider Institute voraus.

Abgesehen von der grundsätzlichen Frage, ob ein „zweites Qualitätsinstitut“ wirklich sinnvoll ist (s.o.), sticht in der neuen Fassung des $\$ 137$ a vor allem die strukturelle Schwäche des neuen Institutes hervor. Der Gesetzgeber hat sehr auf die Analogie zur Struktur des IQWiG gesetzt. Die Institutsleitung wird durch den Vorstand der Stiftung berufen, der wiederum durch den GBA gebildet wird (plus ein Vertreter des BMG). Gleichzeitig sind die GBA-Partner wie KBV, DKG BÄK und Spitzenverband der Krankenkassen bei der „Entwicklung der Inhalte nach Abs. 3 zu beteiligen“ (Abs. 3: Aufgaben des Institutes, s.o.), d.h. im Klartext, dass Auftragsvergabe, Aufsicht, Vorstand und Bestellung der Institutsleitung von den gleichen Institutionen gewährleistet werden sollen, eine aller Wahrscheinlichkeit nach äußerst ungünstige Konstellation (im Falle des IQWiG würde das heißen, die pharmazeutische Industrie wäre sowohl im Vorstand vertreten als auch bei der Auftragsvergabe stimmberechtigt). So erklären sich auch die durchweg positiven Reaktionen der Selbstverwaltungspartner, die darauf hoffen, dass die vergleichende Qualitätsberichterstattung dann nur noch vom neuen Institut betrieben werden darf und die unabhängigen bzw. kassenseitigen Vergleich nicht mehr autorisiert sind (Abs. 3 Nr. 5).

\subsection{Ebene des Nutzens (Allokation und Effizienz)}

Die Frage, ob Patienten mit einer Methode behandelt werden, deren Nutzen für die Patienten belegt ist, stellt einen der zentralen Qualitätsperspektiven dar (s. Kap. 1.3 und 3.3). Eine Methode, deren Nutzen aus wissenschaftlichen sowie fachlichen Kriterien als belegt angesehen wird und die im professionellen Rahmen akzeptiert wird, bedingt den objektiven Bedarf an dieser Methode (SVR 2001, Band III, Nr. 24 und 3off). Von Bedeutung sind die folgenden Aspekte, nach denen der Nutzen bestimmt wird: traditionell die Meinung der Experten, weiterhin die sog. Lehrbuchmeinung (im juristischen Kontext), dann mit Aufkommen der EBM verstärkt der randomisierte Doppelblindversuch im Rahmen der klinisch evaluativen Forschung und letztlich die Versorgungsforschung (Schrappe und Scriba 2006), die die Angemessenheit der Me- 
thode untersucht, kontrolliert in ihrer internen Validität durch Evidence-Based Health Care (Pfaff und Schrappe 2011) (s. Abb. 34).

In den letzten 15 Jahren hat die Nutzenbewertung in Deutschland eine enorme Entwicklung genommen. Durch in Aufnahme der Begriffe „evidenzbasierte Medizin“, „Leitlinien“ und „Versorgungsforschung“ in das 5. Sozialgesetzbuch ist ein umfassender und zu diesem Zeitpunkt einmaliger Versuch gestartet worden, hier zu objektiven und nachvollziehbaren Einschätzungen zu kommen. Die zentrale Institution, die es in ihrer „neokorporatistischen Rolle“ (wenn man das so bezeichnen darf) zur Aufgabe hat, diesen Prozess der Nutzenbewertung verlässlich und kontinuierlich ablaufen zu lassen, ist wiederum der Gemeinsame Bundesausschuss (GBA), der auf der Basis der Verfahrensordnung nach $₫ 91$ Abs . 4 Richtlinien zur ärztlichen und Krankenhausbehandlung beschließt (\$ 92). Dabei lässt er sich durch ein „fachlich unabhängiges, rechtsfähiges, wissenschaftliches Institut für Qualität und Wirtschaftlichkeit im Gesundheitswesen“ (IQWiG) beraten ( $\$ 139 \mathrm{a}-\mathrm{C})$.

Die Einrichtung des IQWiG lehnt sich an internationale Vorbilder (z.B. NICE in Großbritannien) an und wird „zu Fragen von grundsätzlicher Bedeutung für die Qualität und Wirtschaftlichkeit der im Rahmen der gesetzlichen Krankenversicherung erbrachten Leistungen“ tätig (\$139a Abs. 3). Es recherchiert den allgemeinen Wissensstand, erstellt Ausarbeitungen zur Fragen der Qualität und Wirtschaftlichkeit, stellt der Öffentlichkeit Informationen zu Qualität und Effizienz zur Verfügung, bewertet Leitlinien, gibt Empfehlungen zu Disease Management-Programmen ab und bewertet - das ist eine der wichtigsten Aufgaben - Kosten und Nutzen von Arzneimitteln. Das Institut hat dabei „zu gewährleisten, dass die Bewertung des medizinischen Nutzens nach den international anerkannten Standards der evidenzbasierten Medizin und die ökonomische Bewertung nach den hierfür maßgeblichen international anerkannten Standards, insbesondere der Gesundheitsökonomie erfolgt“ (\$139a Abs. 4).

Gerade bei der Bewertung des Nutzens von Arzneimitteln werden die unterschiedlichen Aspekte, unter denen Nutzen beurteilt werden kann, deutlich. Nach $₫ 35$ a „Bewertung des Nutzens von Arzneimitteln mit neuen Wirkstoffen“

$\begin{array}{lll}\text { Wirksamkeit } & \begin{array}{l}\text { klinisch-evaluative Forschung } \\ \text { absolut (efficacy) }\end{array} & \begin{array}{l}\text { Versorgungsforschung } \\ \text { relativ (effectiveness) }\end{array} \\ \text { Nutzenbewertung } & \text { klinische Endpunkte } & \text { Angemessenheit } \\ \text { präferierte Meth. } & \text { RCT >CT > HCT } & \ldots+\text { sozialwiss. Meth. } \\ \text { interne Validität } & \text { EBM } & \text { EBHC/HTA } \\ \text { externe Validität } & \text { niedrig(er) } & \text { hoch (höher) } \\ \text { Bedarf } & & \text { objektiv }\end{array}$

Abb. 34 Klinisch evaluative und Versorgungsforschung bei der Beschreibung des Nutzens und objektiven Bedarfs. RCT randomisierter kontrollierter Versuch, HCT historisch, EBHC Evidence-Based Health Care, HTA Health Technology Assessment 
muss der GBA den Nutzen von erstattungsfähigen Arzneimittel mit neuen Wirkstoffen bewerten, wobei ,insbesondere die Bewertung des Zusatznutzens gegenüber der zweckmäßigen Vergleichstherapie, des Ausmaßes des Zusatznutzens und seiner therapeutischen Bedeutung “ zu beachten ist (Abs. 1 Satz 2). Nur für Arzneimittel für „,seltene Leiden“ gilt der „Zusatznutzen durch die Zulassung als belegt“ (Satz 10), im Übrigen ein Widerspruch in sich, denn bei der Zulassung spielt der Zusatznutzen kaum eine Rolle. Außerdem kann das IQWiG mit einer Kosten-Nutzen-Bewertung beauftragt werden ( $\$ 35 \mathrm{~b}$ Abs. 1), beim „Patientennutzen sollen insbesondere die Verbesserung des Gesundheitszustandes, eine Verkürzung der Krankheitsdauer, eine Verlängerung der Lebensdauer, eine Verringerung der Nebenwirkungen sowie eine Verbesserung der Lebensqualität, bei der wirtschaftlichen Bewertung auch die Angemessenheit und Zumutbarkeit einer Kostenübernahme durch die Versichertengemeinschaft, angemessen berücksichtigt werden. "Neben den klassischen Endpunkten wie Verlängerung der Überlebenszeit wird hier auf die Verringerung der Nebenwirkungen und die Verbesserung der Lebensqualität abgehoben, vor allem letzteres ein Kriterium aus dem Bereich der Nutzenbestandteile, die von Patienten direkt angegeben (Patient-Reported Outcome Measures) und im Rahmen von Versorgungsforschungsstudien erhoben werden. Solche „Versorgungsstudien“ - das ist der etwas unbeholfene Ausdruck des Gesetzgebers für Versorgungsforschungsstudien (s. auch Koalitionsvertrag vom 23.11.2013) - können nach Abs. 2 mit dem „pharmazeutischen Unternehmer“ vereinbart werden, wenn es zusätzlichen Klärungsbedarf gibt.

Wir stehen in Deutschland also vor der Situation, dass durch einen länger dauernden, in mehreren Schritten ablaufenden Gesetzgebungsprozess nicht nur die klassischen Nutzenbelege aus der klinisch evaluativen Forschung (Ergebnisse kontrollierter klinischer Studien und deren Synthese [Systematische Reviews, Metaanalysen]) in den Allokationsentscheidungen einbezogen werden, sondern man hat eine Institution geschaffen (GBA), die diesen Allokationsprozess organisiert und sich dabei fachlich wissenschaftlich durch das IQWiG beraten lassen kann. Man hat außerdem begonnen, sogar weitergehende Nutzenbestand teile zu adressieren (pars pro toto: Lebensqualität), die der Sachverständigenrat unter dem Begriff der Angemessenheit zusammengefasst hat (SVR 2008, Nr. 579). Letztendlich kann man damit rechnen, dass es als Ergänzung der Nutzenbewertung auf der Basis „klassischer“ klinischer Studien zur Durchführung eines Nutzenreviews für jede wichtige Methode kommt, die im Spektrum der Angemessenheit eine Rolle spielt. Es handelt sich dabei um ein Themenspektrum, das auch von den Health Technology Assessment (HTA)-Berichten abgedeckt wird. Dies wäre anzustreben; denn was ist die beste Methode wert, wenn sie sich in der Gesellschaft, im professionellen Rahmen, bei den Patienten aus irgendwelchen Gründen, die z.B. in den Präferenzen oder in finanziellen Beweggründen liegen, nicht durchsetzen kann. Leider ist die HTA-Gruppe im DIMDI noch immer nicht aus ihrem Schattendasein hinausgetreten. 


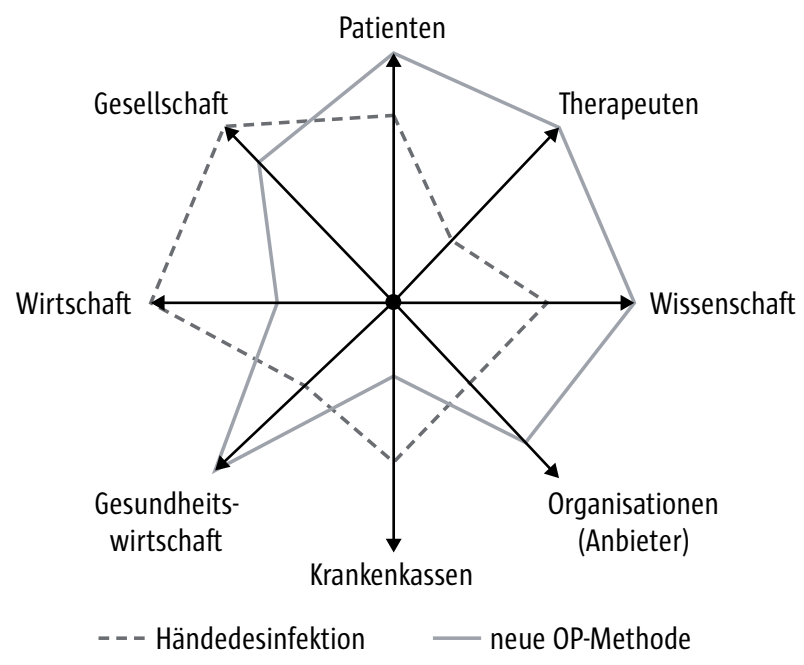

Abb. 35 Perspektiven zur Beurteilung des Nutzens von Versorgungsleistungen

Allerdings ist die Auseinandersetzung über die Nutzenbestandteile nicht einfach (auch wenn sie gut organisiert im CBA ablaufen sollte), nicht nur deswegen, weil es unterschiedliche Aspekte gibt (z.B. können Präferenzen aus sozialen oder ökologischen Beweggründen solchen aus finanzieller Sicht widersprechen), sondern weil es unterschiedliche Perspektiven gibt, nach denen die Nutzenbestandteile bewertet werden. Ähnlich wie die 6 Perspektiven, die in diesem Gutachten für die Darstellung von Qualität verwendet werden (s. Kap. 1.3), kann man hier (natürlich stark vereinfachend, trotzdem schon sehr komplex) acht solcher Perspektiven unterscheiden (s. Abb. 35). Wichtig ist es, zum Beispiel der Perspektive der Gesundheitsberufe die der Gesundheitsinstitutionen an die Seite zustellen, genauso zwischen der gesellschaftlichen und der Perspektive der Krankenkassen zu unterscheiden (dies wird in Zukunft wichtig sein, wenn Leistungserbringung und Versicherungsfunktion mehr ineinanderfließen), und letztlich ist auch nicht in jedem Fall das Interesse der Gesundheitswirtschaft deckungsgleich mit dem Interesse der Gesamtwirtschaft. Beispielhaft sind in Abbildung 35 zwei Methoden, nämlich die Händedesinfektion zur Vermeidung nosokomialer Infektionen, und eine hypothetische neue Operationsmethode, aufgetragen. Man erkennt leicht an diesem Beispiel, dass je nach Perspektive der Nutzen dieser Maßnahmen sehr unterschiedlich beurteilt wird, woraus zwei sehr unterschiedliche Muster entstehen.

\subsection{Patientenperspektive}

Das (zynische) Bonmot, der Patient stehe im Mittelpunkt, dort aber im Weg, hat nicht umsonst so viele Auflagen erlebt. Paul M. Ellwood hat es in seiner Shattuck Lecture im Jahr 1988 höflicher formuliert: 
"We acknowledge that out common interest is the patient, but we represent that interest from such divergent, even conflicting, viewpoints that everyone looses perspective" (Ellwood 1988)

Vor voreiligen Lösungen ist also zu warnen. Vielleicht ist es auch heilsam, zunächst erst einmal darauf zu verweisen, dass die Patientenperspektive nicht die einzige Perspektive darstellt, mit der man das Gesundheitswesen betrachten kann; es ist die wichtigste, aber es gibt genauso die Perspektive der Institutionen, der Gesellschaft, der Gesundheitsberufe etc. Und so ist in diesem Gutachten die Patientenperspektive eine von sechs Qualitätsperspektiven (s. Kap. 1.3) - und eine von acht Nutzenperspektiven (s. Kap. 7.3). In Kapitel 2.4.7 wurde darauf verwiesen, dass Qualitätsdaten auf der Basis von Patientenerfahrungen (Patient-Reported Outcome Measures) eine wichtige Rolle als Indikatoren spielen (und jetzt auch in der Arbeit des „Institutes für Qualitätssicherung und Transparenz im Gesundheitswesen“ Beachtung finden sollen). In Kapitel 3.4 werden die derzeitigen Qualitätsdefizite aus der Perspektive der Patienten thematisiert und in Kapitel 4.4 bzw. 5.3 (Public Reporting) der Qualitätswettbewerb mit seiner These der Nutzenmaximierung und Aufhebung der Informationsasymmetrie dargestellt.

Der Blick in die gesetzlichen Grundlagen und Entwicklungen der letzten Jahre zeigt, dass hier Einiges angekommen ist bzw. angestoßen werden konnte. Die Entwicklung verlief zweigeteilt: einerseits kam es zu einer besseren Repräsentation der Patienten in den Institutionen des Gesundheitssystems (vor allem in den Gremien, die sich mit Allokationsfragen und Qualitätssicherung beschäftigen), andererseits hat man versucht, mit dem Patientenrechtegesetz vom 11.1.2013 die haftungsrechtliche Situation der Patienten zu konsolidieren, unter gleichzeitiger Förderung von Präventionsmaßnahmen zur Verbesserung der Patientensicherheit.

Ganz allgemein sind nach $\$ 140$ of Abs. 1 ,die für die Wahrnehmung der Interessen der Patientinnen und Patienten und der Selbsthilfe chronisch kranker und behinderter Menschen maßgeblichen Organisationen in Fragen, die die Versorgung betreffen“ zu beteiligen. Dies bezieht sich vor allem auf die Tätigkeit des GBA, wo Patientenvertreter (auch bei Beschlussfassung) anwesend sind und Anträge stellen können (Abs. 2), aber auch auf die Unabhängige Institution nach 137a Abs. 3 zur sektorenübergreifenden Qualitätssicherung bzw. dessen Nachfolgeorganisation, dem „Institut für Qualitätssicherung und Transparenz im Gesundheitswesen“. In $\mathbb{1} 135 \mathrm{a}$ Abs. 2 wird ein „Patientenorientiertes Beschwerdemanagement“ in Krankenhäusern als Teil des Qualitätsmanagements vorgeschrieben.

Diese Beteiligung wirkt sich auch in einer allgemein stärkeren Aktivität von Patientenvertretern und -verbänden in der Gesundheitspolitik und gesundheitspolitischen Diskussion aus. Patientenvertreter haben z.B. im Jahr 2005 das Aktionsbündnis Patientensicherheit e.V. mit gegründet, auf wissenschaftlichen Kongressen wird die Stimme der Patientenvertreter gehört, auch die 
Pharmaindustrie ist sich über die Bedeutung dieser Verbände bzw. Selbsthilfeverbände im Klaren.

Das Patientenrechtegesetz von Anfang 2013 hatte einen anderen Impetus. Dieser sich über lange Jahre hinziehende Gesetzgebungsprozess fußte auf der allgemein verbreiteten Ansicht, die rechtliche Situation der Patienten sei zu wenig übersichtlich gestaltet, und die zahlreichen, durch die fortlaufende Rechtsprechung normierten Regelungen seien in ihrer Menge kaum für die Patientenseite handhabbar. Diesem Eindruck kann man wenig entgegensetzen. Das Kernstück dieses Gesetzes besteht dann auch in der Einführung des Vertragstypus „Behandlungsvertrag“, der nach $\$ 6300 \mathrm{ff}$. BGB dem Dienstvertrag an die Seite gestellt wird. Man möge dem Autor dieses Gutachtens verzeihen, wenn hier aus Sicht eines Nicht-Juristen einige Anmerkungen gemacht werden:

- Bezüglich mehrerer Sachverhalte ist es tatsächlich zu einer Klärung von strittigen Punkten gekommen, so ist z.B. in $\$ 630 e$ Abs. 1 . Satz 3 die in der Praxis immer noch vernachlässigte Pflicht zur Aufklärung von Behandlungsalternativen klargestellt: „Bei der Aufklärung ist auch auf Alternativen zur Maßnahme hinzuweisen, wenn mehrere medizinisch gleichermaßen indizierte und übliche Methoden zu wesentlich unterschiedlichen Belastungen, Risiken oder Heilungschancen führen können. “

- Im SGB V ist (wie oben bereits dargestellt) in $\mathbb{} 137$ ein Absatz 1 d eingefügt worden mit dem Inhalt, dass der GBA „Maßnahmen zur Verbesserung der Patientensicherheit“ und „insbesondere Mindeststandards für Risikomanagement- und Fehlermeldesysteme“ festlegen soll. Gleichermaßen wurde in Abs. 135a SGB V der Hinweis aufgenommen, dass CIRS-Meldungen nicht zum Nachteil der Meldenden verwendet werden dürfen, es sei denn es handelt sich um eine Straftat, die „im Höchstmaß mit mehr als fünf Jahren Freiheitsstrafe bedroht ist “ (zur Einschätzung s. hierzu Kap. 7.2.1).

- eine Klarstellung hat es auch hinsichtlich der Kommunikation im Schadensfall gegeben. Hintergrund dieser Regelung ist die Forderung, behandelnde Ärzte sollten sich auf bei Auftreten eines Schadens beim Patienten entschuldigen, sich also zu einem Fehler mit Schadensfolge bekennen; ein solches Verfahren ist einerseits ein wichtiger Schritt zur allgemeinen Vertrauenskultur, man weiß aber auch, dass durch eine Entschuldigung das Risiko einer gerichtlichen Auseinandersetzung deutlich abnimmt. Unklar waren allerdings die haftungs- und versicherungsrechtlichen Konsequenzen, von daher ist die Regelung in $\mathbb{5} 630 \mathrm{C}$ Abs. 2 Satz 3 BGB sinnvoll: „Ist dem Behandelnden oder einem seiner in $\mathbb{} 52$ Absatz 1 der Strafprozessordnung bezeichneten Angehörigen ein Behandlungsfehler unterlaufen, darf die Information nach Satz 2 zu Beweiszwecken in einem gegen den Behandelnden oder gegen seinen Angehörigen geführten Straf- oder Bußgeldverfahren nur mit Zustimmung des Behandelnden verwendet werden.“ Der genannte „Satz 2“ beschreibt 
die Entschuldigungssituation mit den Worten: „Sind für den Behandelnden Umstände erkennbar, die die Annahme eines Behandlungsfehlers begründen, hat er den Patienten über diese auf Nachfrage oder zur Abwendung gesundheitlicher Gefahren zu informieren." Nach Ansicht des Autors dieser Zeilen war dies zumindest für den Fall der Abwendung von gesundheitlichen Gefahren, also im Falle therapeutischer Konsequenzen, schon zuvor in der Rechtsprechung festgeschrieben.

- Keine Änderung ergaben sich hinsichtlich sonstiger Aspekte der Aufklärung ( $\$ 630 e$ Abs. 2), denn die Rechtsprechung war hinsichtlich der Person, die die Aufklärung durchzuführen hat, und hinsichtlich des Zeitpunkts (keine absolute Angabe, sondern auf die Möglichkeit einer „wohlüberlegten "Entscheidung) durchgängig zu derselben Ansicht gekommen.

- Gleiches gilt für die „Berichtigungen und Änderungen von Eintragungen in die Patientenakte“ ( $\$ 63$ of Abs. 1 BGB) und die Einsichtnahme in die Patientenakte ( $\$ 630$ g Abs. 1 BGB), wobei die Einschränkung, dass „der Einsichtnahme nicht erhebliche therapeutische Gründe oder sonstige erhebliche Rechte Dritter entgegenstehen“ dürfen, schon Gewicht hat.

- Zu der wichtigen Thematik der Beweislasterleichterung wird in $\mathbb{5} 630 \mathrm{oh}$ BCB Stellung genommen, denn im Vorfeld der Gesetzgebung war eine grundsätzliche Abkehr von der gängigen Praxis in der Diskussion dass die primäre Beweislast beim geschädigten Patienten liegt. Hier wird aber bezüglich der voll beherrschbaren Risiken, der Dokumentationspflichten, des Übernahmeverschuldens und des groben Behandlungsfehlers die bisherige Linie weitergeführt.

\subsection{Professionelle Perspektive}

Zusätzlich zu den Bestimmungen der $\$ 135 a$ und 137 SGB V werden in $\$ 136$ die Kassenärztlichen Vereinigungen in ihrer Sicherstellungsfunktion für die ambulante Versorgung in die Pflicht genommen. „Die Kassenärztlichen Vereinigungen haben Maßnahmen zur Förderung der Qualität der vertragsärztlichen Versorgung durchzuführen“ ( $\$ 136$ Abs. 1), die Ergebnisse sind zu veröffentlichen. Der GBA hat nach $\$ 92$ Abs. 1 Satz 2 Nr. 13 eine Richtlinie zu erstellen, in der „Kriterien zur Qualitätsbeurteilung in der vertragsärztlichen Versorgung“ ( $\$ 136$ Abs. 1) niedergelegt sind. Diese Richtlinie „über grundsätzliche Anforderungen an ein einrichtungsinternes Qualitätsmanagement für die an der vertragsärztlichen Versorgung teilnehmenden Ärzte, Psychotherapeuten und medizinischen Versorgungszentren“( GBA 2014B) hebt z.B. nach $₫ 3$ für die Patientenversorgung die drei Aspekte "Ausrichtung der Versorgung an fachlichen Standards und Leitlinien“, die „Patientenorientierung, Patientensicherheit, Patientenmitwirkung, Patienteninformation und -beratung“ sowie die „Strukturierung von Behandlungsabläufen " hervor. Weiterhin werden ein Hygienemanagement, Maßnahmen 
zur Risiko- und Fehlermanagement und zum Beschwerdemanagement verlangt.

Jenseits der gesetzlichen Ebene ist die Entwicklung im professionellen Bereich höchst widersprüchlich. Unter Einbeziehung des Konzeptes der Expertenorganisation (s. Kap. 8.2) und des Professionalismus (s. Kap. 8.4.3) wird im Rahmenkonzept hierzu hinsichtlich der Frage, wie die weitere Entwicklung einzuschätzen ist, Stellung genommen (s. Kap. 9). In jedem Fall war es eine der erstaunlichsten Erfahrungen der letzten Jahre, die positive Resonanz der professionellen Einrichtungen im Gesundheitswesen (über alle Berufsgruppen hinweg) auf die Thematisierung der Problematik Patientensicherheit zu beobachten. Man hat viele Theorien diskutiert, warum diese Entwicklung so günstig verlaufen ist. Am wahrscheinlichsten erscheint dabei die Annahme, dass die Möglichkeit, dieses dringende Problem endlich angehen zu können („über Fehler sprechen lernen“, s.a. die damalige Broschüre „Aus Fehlern lernen“ des Aktionsbündnis Patientensicherheit e.V.) derart entlastend wirkte, dass die Befürchtungen vor Gesichts- bzw. Ansehensverlust oder Sanktionen zurückstehen konnten.

Auch die Selbstverwaltung und deren Institutionen schlossen sich den Initiativen an, die Arbeitsgruppen des Aktionsbündnisses Patientensicherheit (APS) zu unterschiedlichen Themen waren (berufsgruppenübergreifend) gut besucht, und die Ergebnisse konnten sich sehen lassen. Insbesondere die Deutsche Krankenhausgesellschaft und die Bundesärztekammer unter ihrem damaligen Präsidenten Prof. Hoppe waren sehr aktiv, außerdem die gesetzlichen Krankenkassen (bes. AOK, TK) und natürlich zahlreiche Einzelpersonen. Es gab Preisverleihungen (z.B. Berliner Gesundheitspreis), auf den jährlich stattfindenden Kongressen der GQMG, des DNVF und des APS (um nur einige zu nennen) war das Thema auf der Tagesordnung. Dies gilt und galt auch für den Deutschen Ärztetag, der sich im Jahr 2005 schwerpunktmäßig mit diesem Thema beschäftigte, und z.B. dem Jahreskongress der Deutschen Gesellschaft für Chirurgie, die unter ihrem damaligen Präsidenten Prof. Rothmund, seinerseits Gründungsmitglied des APS und Vorstandmitglied der ersten Stunde, im gleichen Jahr dieses Thema gut sichtbar (und hoch umstritten) aufgriff. Es bildeten sich überinstitutionelle CIRS-Arbeitsgruppen so wie das CIRS-Netz der Anästhesie (Berufsverband Deutscher Anästhesisten), dem CIRS der Deutschen Gesellschaft für Chirurgie und dem Krankenhaus-CIRS-Netz Deutschland (neben dem APS und der DKG getragen von dem Ärztlichen Zentrum für Qualität (KBV, BÄK) und dem Deutschen Pflegerat). Auch auf dem Gebiet der Schiedsstellen der Landesärztekammern tat sich vieles, man arbeitete enger zusammen und fing an, die dort eingegangenen und begutachteten Fälle von vermuteten Behandlungsfehlern mit der Absicht zu analysieren, Muster für die Konstruktion von Präventionsmaßnahmen zu finden. Man kann aus diesen Zahlen zwar keine quantitativen Angaben über die Häufigkeit von unerwünschten Ereignissen ableiten (s. Kap. 2.2), aber es ist sehr gut möglich, 
diese Daten als „generierende Methoden“ zur Identifikation von vorher nicht bekannten Risikosituationen zu verwenden.

Diese positive Erfahrung stand im Widerspruch zur Erfahrung mit der Einführung der Qualitätssicherung bzw. des Qualitätsmanagements in den goer-Jahren des letzten Jahrhunderts, wenngleich auch gesagt werden muss, dass die Fachgruppen der externen Qualitätssicherung eine große Akzeptanz gefunden haben (z.B. neonatale Qualitätssicherung). In der Breite gesehen hat es eine große Menge von Qualitätsinitiativen aus dem professionellen Bereich gegeben, die sich mit Qualität durchaus selbstkritisch beschäftigt haben und immer noch beschäftigen. An dieser Stelle sollten auch die vielfältigen Fortbildungsangebote zu Qualitätsmanagement, Risikomanagement, evidenzbasierte Medizin und Ärztliche Führung Erwähnung finden, die seit den goer-Jahren von den Landesärztekammern angeboten werden.

Es ist zu hoffen, dass von der professionellen Seite auch in Deutschland vermehrt Aktivitäten ausgehen, die sich z.B. mit der amerikanisch/kanadischen Choosing wisely-Initiative vergleichen lassen (s. Kap. 3.3.1).

\subsection{Institutionelle Perspektive}

Auf das institutionelle Qualitäts- und Risikomanagement wurde bereits in Kapitel 4.3 ausführlich eingegangen, zu den Regelungen nach $\mathbb{1} 135 \mathrm{a}, \mathbb{1} 137$ und $\$ 137$ a (s. Kap. 7.2). Grundsätzlich sind Institutionen im Gesundheitswesen als Expertenorganisationen externen Qualitätsanforderungen gegenüber schwer zugänglich (s. Kap. 8.2), wichtig ist es also, Bedingungen zu schaffen, die die interne Umsetzung fördern und fordern. Der Gesetzgeber tut dies im Bereich der Qualitätssicherungsergebnisse und auch der Infection Control Thematik z.B. dadurch, dass er die leitenden Ärzte „verpflichtet, die Ergebnisse, Vergleiche und Bewertungen, die ihr oder ihm aus der Qualitätssicherung zugeleitet werden, mit allen Mitgliedern der Krankenhausleitung und den unmittelbar am Behandlungsprozess beteiligten Mitarbeiterinnen und Mitarbeitern anhand der Ergebnisse der Qualitätssicherungsmaßnahme durchzusprechen, kritisch zu analysieren und ggf. notwendige Konsequenzen festzulegen." (\$ 3 Abs. 2 der QSKH-RL [GBA 2014A]), über diese Gespräche ist ein Nachweis zu führen (Abs. 3). Diese Vorschriften können durchaus einen organisatorischen Wandlungsprozess anstoßen, ähnlich wie das Public Reporting (s. Kap. 5) oder zukünftige Pay for Performance Projekte (s. Kap. 6) diese Option besitzen.

Die Deutsche Krankenhausgesellschaft (DKG) als Dachorganisation der deutschen Krankenhäuser hat am 9.4.2014 ein Positionspapier zur Qualitätssicherung und Patientensicherheit beschlossen (DKG 2014), das teilweise eine sehr aktive Stellung zur Problematik einnimmt, teilweise aber auch vor allem hinsichtlich der Forderung, dass Qualitätssicherung gesondert finanziert werden müsse, kritisch zu sehen ist. Sehr zu begrüßen sind die Vorstellungen insbe- 
sondere zur Verbesserung der Patientensicherheit (Fehlermeldesysteme, Patientenidentifikation, Checklisten, Arzneimitteltherapiesicherheit und Hygiene). Bei aller Widersprüchlichkeit in der öffentlichen Positionierung war die DKG z.B. Gründungsmitglied der „Kooperation für Transparenz und Qualität im Gesundheitswesen“ (KTQ) und auch des Aktionsbündnis Patientensicherheit e.V., hat die Qualitätsproblematik also aktiv mitgestaltet. Bei der Erarbeitung der APS-Empfehlungen z.B. zur Seiten- und Eingriffsverwechselung hat die DKG nicht nur inhaltlich mitgearbeitet, sondern auch für eine Disseminierung der Empfehlung in die deutschen Krankenhäuser möglich gemacht. In einer vom APS durchgeführten Umfrage zum Stand des klinischen Risikomanagements in 484 deutschen Krankenhäusern > 50 Betten (Rücklaufquote $27 \%$ von 1.815 angefragten Häusern) gaben bereits im Jahr $2008 \mathrm{ca}$. ein Viertel bis ein Drittel der Krankenhäuser an, unterschiedliche Maßnahmen zum klinischen Risikomanagement ergriffen zu haben (Lauterberg et al. 2012).

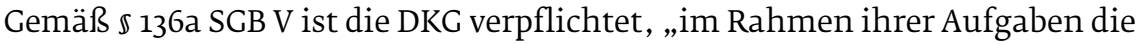
Qualität der Versorgung im Krankenhaus“ zu fördern. Interessanterweise, dies am Rande, ist hier ein einem der „Qualitätsparagraphen“ des SGB V wiederum versucht worden, die unerwünschten Anreize des dominierenden Vergütungssystems, in diesem Fall des DRG-Systems, durch Qualitätsvorgaben auszugleichen, indem ,im Einvernehmen mit der Bundesärztekammer Empfehlungen abzugeben [sind], die sicherstellen, dass Zielvereinbarungen, die auf finanzielle Anreize bei einzelnen Leistungen abstellen, ausgeschlossen sind“ ( $\$ 136 a$ Satz 2). Es soll also ausgerechnet auf der Ebene der arbeitsvertraglichen Regelungen auf Chefarztebene der Mengenanreiz ausgeglichen werden, der wichtigste unerwünschte Effekt eines fallpauschalierenden Vergütungssystems. Dies ist ein gutes Beispiel für einen nicht sachgerechten, defensiven Einsatz von Qualitätsinstrumenten, die kaum jemals in der Lage sind, die dominierende Anreizstruktur des Vergütungssystems zu neutralisieren (s. Kap. 4.1).

Gleichwohl ist es gelungen, institutionell nützliche Regelungen in den Gesetzgebungsprozess einfließen zu lassen. So konnte in $₫ 137$ Abs. 3 aufgenommen werden, dass „Meldungen und Daten aus einrichtungsinternen und einrichtungsübergreifenden Risikomanagement- und Fehlermeldesystemen (...) im Rechtsverkehr nicht zum Nachteil des Meldenden verwendet werden“ dürfen. Dies gilt zwar nicht bei vermuteten schwerer wiegenden Straftaten, trotzdem ist dies für die Einrichtung eines CIRS-Systems oder anderer „generierender Verfahren" ein wichtiger Baustein, der für Beruhigung sorgt (zur Wertung s. Kap. 7.2).

Es hat in der Vergangenheit eine ganze Reihe von erfolgreichen und zukunftsfähigen institutionellen Initiativen gegeben, die den Qualitätsgedanken durchaus auch zur Abgrenzung zur Konkurrenz in den Vordergrund stellen und sich für Transparenz aussprechen. Im ambulanten Bereich gibt es zahlreiche Ärzte- bzw. Versorgungsnetze, als Paradebeispiel kann die Initiative 
„Gesundes Kinzigtal“ gelten. Auch hat die Private Krankenhausindustrie hat qualitätsbezogene Aktivitäten entwickelt, wie z.B. die Initiative Qualitätsmedizin (IQM) mit ihrem Peer Review Verfahren (Helios-Kliniken) oder die Initiative Qualitätkliniken.de der Sana-Kliniken.

\subsection{Wissenschaft}

Die wissenschaftliche Perspektive, die sechste ihrer Art nach Kapitel 1.3, wird vom Gesetzgeber nur am Rande ins Spiel gebracht. Allerdings bleibt die Wissenschaft auch hier nicht ungehört, so sind z.B. die Arbeitsgemeinschaft Wissenschaftlich-Medizinischer Fachgesellschaften (AWMF) und das Deutsche Netzwerk Versorgungsforschung (DNVF) in den Regelungen zum neuen „Institut für Qualitätssicherung und Transparenz im Gesundheitswesen“ angesprochen, das Bestandteil des Finanzstruktur- und Qualitätsweiterentwicklungsgesetzes (FQWG) war (s. Kap. 7.2.3). Nach $\mathbb{S} 137$ a Abs. 7 ist beiden Einrichtungen aufgetragen, sich an der „Entwicklung der Inhalte“ nach Abs. 3 $\mathrm{zu}$ beteiligen, womit die Gestaltung der zahlreichen Aufgaben gemeint ist, die von diesem neuen Institut erledigt werden sollen.

Weiterhin sind die AWMF, die wissenschaftlichen Fachgesellschaften ganz allgemein, und die Fachgesellschaften, die Mitglied im DNVF sind, im Besonderen, außerdem die Gesellschaft für Qualitätsmanagement in der Gesundheitsversorgung (GQMG) und Andere natürlich aktiv in der Forschung und Gestaltung der Qualität des Gesundheitswesen. Allerdings ist es ja landläufig bekannt, dass eine derartige Forschung durch die noch immer nicht hinreichende Repräsentation der Versorgungsforschung in den Medizinischen Fakultäten immer noch nicht einen adäquaten Stellenwert hat, auch wenn vonseiten der Forschungsförderung (BMG, BMBF, DFG u.a.) in den letzten Jahren hier einiges getan wurde. Die Förderinitiative der Bundesärztekammer zur Versorgungsforschung ist als lobenswertes Beispiel herauszuheben.

\subsection{Gesetzliche Regelungen: Zusammenfassung}

- Die gesetzlichen Regelungen zu Qualität und Patientensicherheit in Deutschland weisen eine bemerkenswerte Regelungstiefe auf und sich von fast unübersehbarer Vielfalt. Sie regeln zahlreiche Detailprobleme wie die Einführung von Fehlermeldesystemen (Risikomanagement) und Hygienemaßnahmen. Ein Schwerpunkt liegt auf den institutionellen Regelungen, auch wurden die Rechte der Patienten und Patientenverbände gestärkt (Patientenrechtegesetz). Die professionelle und wissenschaftliche Perspektive lassen sich naturgemäß durch normative Vorgaben wenig beeinflussen, aber auch hier wurde durch die Einrichtung des Gemeinsamen Bundesausschusses eine Institutionalisierung erreicht und somit auch eine gewisse Öffnung der Mesoebene geschaffen. 
- Allerdings existieren deutliche Defizite in der Kohärenz der Regelungen und in der Stringenz einer Entwicklungsperspektive. Zwar sind viele Regelungen im 9. Abschnitt des 5. Kapitels des SGB V unter „Sicherung der Qualität der Leistungserbringung " enthalten, insgesamt sind sie jedoch auf eine derart große Zahl von anderen gesetzlichen Regelungen, Verordnungen und untergesetzlichen Richtlinien wie denen des GBA verteilt, dass kaum ein Überblick möglich ist. Eine übergeordnete und ordnende Botschaft ist dadurch nicht sichtbar, es entsteht der Eindruck, dass keine tragende Entwicklungsperspektive vorhanden ist. Hinzu kommen sachliche Defizite, so wird der transsektorale Auftrag zwar sehr betont, aber es wird nicht beachtet, dass das Ziel eigentlich area-Indikatoren für die Versorgung von Regionen bzw. der Bevölkerung heißen müsste. Auch fehlt eine Bezugnahme auf die Handlungsnotwendigkeiten der Zukunft (Alterung, Versorgung in ländlichen Regionen mit geringer Bevölkerung, Multimorbidität, Chronizität). Die externe Qualitätssicherung, die sich historisch aus den Fallpauschalen des Gesundheitsstrukturgesetzes von 1992 ableitet, umfasst fast ausschließlich operative Akuterkrankungen.

Durch die Dualität des Institutes für Qualität und Wirtschaftlichkeit im Gesundheitswesen (IQWiC) und des neuen Institutes für Qualitätssicherung und Transparenz im Gesundheitswesen (IQTiC) wird die Aufspaltung des Qualitätsthemas in Qualitätsdarstellung und Nutzen von Behandlungsmethoden festgeschrieben. Der inhaltliche Schwerpunkt der Regelungen zu Qualität und Patientensicherheit liegt eindeutig in den Perspektiven gesellschaftliche Qualität (unter Einbeziehung der klassischen Qualitätssicherung nach $\$ 137$ SGB V) und der Bewertung des Nutzens (Effizienz) von Behandlungsmethoden (in der ersten Linie Arzneimitteln). Es handelt sich hierbei um die zwei zentralen Aufgaben des GBA, der sich wissenschaftlich durch die zwei genannten wissenschaftlichen Instituten beraten lässt, die schon durch ihre Namensgebung (beide führen „Qualität“ im Namen) die Frage aufwerfen, aus welchem Crund hier ein einziges Institut nicht ausreicht. In Großbritannien liegt die Entwicklung der Indikatoren für das QOF-Programm, das die gesamte ambulante Versorgung des NHS umfasst, in den Händen des NICE, der entscheidenden wissenschaftlichen Beratungsinstanz in Großbritannien für Allokationsfragen und eines der weltweit führenden Institute im Bereich Evidence-Based Medicine und Versorgungsforschung. Beim IQTiC kommen schwerwiegende Zweifel an der Funktionsfähigkeit wegen der unklaren Aufteilung von Auftragsvergabe, Aufsicht und operativer Umsetzung hinzu. 